Yanlei Li*, Jian Chen and Songyuan Dai

\title{
Impurity Distribution after Solidification of Hypereutectic Al-Si Melts and Eutectic Al-Si Melt
}

https://doi.org/10.1515/htmp-2018-0117

Received July 18, 2018; accepted October 02, 2018

\begin{abstract}
Hypereutectic Al-Si melts and eutectic Al-Si melt were solidified to study boron and phosphorus distributions in primary silicon phase, eutectic silicon phase and eutectic aluminum phase during Al-Si solvent refining. The boron and phosphorus contents in the primary silicon phase and the eutectic silicon phase were determined by ICP-OES, and the boron and phosphorus contents in the eutectic aluminum phase were calculated by the principle of mass conservation. The primary silicon phase has lowest boron and phosphorus contents, while the eutectic aluminum phase has highest boron and phosphorus contents.
\end{abstract}

Keywords: silicon purification, Al-Si alloy, impurity distribution

JEL Classification: $81.10 . \mathrm{Fq}$

\section{Introduction}

Photovoltaic modules have been installed in many countries, and it is an effective method for protecting environment. Silicon-based solar modules dominate photovoltaic market, and solar grade silicon is the raw material for silicon-based solar modules. The solar grade silicon needs high purity, and it is refined from metallurgical grade silicon. The traditional processes to purify silicon use chemical methods [1], which change the metallurgical grade silicon into compounds, such as $\mathrm{SiHCl}_{3}$ and $\mathrm{SiH}_{4}$, purify these compounds, and reduce them into high purity silicon. These processes have complicated procedures and high energy consumption. New methods with simple procedures and low cost to purify silicon, such as via metallurgical processes, are needed.

*Corresponding author: Yanlei Li, Energy-Saving Building Materials Collaborative Innovation Center of Henan Province, Xinyang Normal University, Xinyang 464000, P. R. China, E-mail: Idymyy@163.com Jian Chen, Institute of Applied Technology, Hefei Institutes of Physical Science, Chinese Academy of Sciences, Hefei 230031, P. R. China

Songyuan Dai, State Key Laboratory of Alternate Electrical Power System with Renewable Energy Sources, North China Electric Power University, Beijing 102206, P. R. China
When the metallurgical grade silicon is purified by the metallurgical processes, boron and phosphorus are difficult to remove because they have high segregation coefficients (value of 0.8 and 0.35 ) in silicon [2]. The solidification of hypereutectic Al-Si melt can remove boron and phosphorus efficiently, because their segregation coefficients in Al-Si melt are smaller than that in silicon [3-8].

After the solidification of hypereutectic Al-Si melt, there are three kinds of phases existed in theory, primary silicon phase, eutectic silicon phase and eutectic aluminum phase. They form in different stages in the solidification process. The primary silicon phase is the product of uniform grain growth in the early stage. The eutectic silicon phase and the eutectic aluminum phase form in the eutectic reaction in the final solidification stage. The boron and phosphorus contents in the primary silicon phase have been studied by many researchers [9-14]. But the boron and phosphorus contents in the eutectic silicon phase and the eutectic aluminum phase have never been studied, and it is investigated in this work.

In addition, many researchers, such as K. Morita [3-5], K. Tang [6], L. Zhao [7], L. Hu [8] studied the boron and phosphorus segregation coefficients in the Al-Si melt, and found that the boron and phosphorus segregation coefficients in the Al-Si melt decrease with decreasing temperature. It means that the boron and phosphorus removal rates increase with decreasing temperature in hypereutectic Al-Si melt. The lowest temperature in the hypereutectic Al-Si melt is the eutectic point temperature, and the eutectic reaction happens at this temperature [15]. The boron and phosphorus removal rates at this temperature have never been studied, and it is investigated in this work.

In this work, two series of experiments were carried out to clarify the boron and phosphorus distributions in the primary silicon phase, the eutectic silicon phase and the eutectic aluminum phase. Hypereutectic Al-Si melts $(\mathrm{Al}-20 \% \mathrm{Si})$ were solidified at different cooling rate, and the boron and phosphorus distributions in the primary silicon phase, the eutectic silicon phase and the eutectic aluminum phase are discussed. Eutectic Al-Si melt (Al$12.6 \% \mathrm{Si}$ ) was solidified at relatively small cooling rate, and the boron and phosphorus distributions in the eutectic silicon phase and the eutectic aluminum phase are discussed. 


\section{Experimental}

The raw materials used in this work are industrial grade aluminum and metallurgical grade silicon, and impurities contents in them determined by inductively coupled plasma optical emission spectrometry (ICP-OES) are shown in Table 1.

Table 1: Impurities contents in the raw materials (ppmw).

\begin{tabular}{lrrrrrrr}
\hline Impurity element & B & P & Al & Fe & Ca & Cu & Mn \\
\hline In silicon & 7 & 11 & 791 & 1357 & 47 & 12 & 64 \\
In aluminum & 7 & 35 & Bal. & 9279 & 113 & 7161 & 2907 \\
\hline
\end{tabular}

\section{The boron and phosphorus distributions after the solidification of hypereutectic Al-Si melts}

The raw materials were prepared for $\mathrm{Al}-20 \% \mathrm{Si}$, which means that it is a hypereutectic Al-Si alloy. As summarized in Figure 1, the raw materials (approximate $250 \mathrm{~g}$ ) were put in an alumina crucible (I.D.: $55 \mathrm{~mm}$, and depth: $100 \mathrm{~mm}$ ). The alumina crucible was put in a resistance heating furnace with a proportional-integral-derivative (PID) controller to control temperature. The furnace was heated to $1071 \mathrm{~K}$ (100 K above the liquidus temperature of $\mathrm{Al}-20 \% \mathrm{Si}$ ) in the atmosphere of argon, and the alloy melted. After that, the melt was stirred by a quartz rod to mix it completely, and cooled to $830 \mathrm{~K}$ ( $20 \mathrm{~K}$ below the eutectic temperature) to solidify it into an ingot with the cooling rate shown in Table 2. After that, the sample was furnace cooled to room temperature.

The solidified ingot was cut into two parts along its vertical axis. The cutting surface of one part was polished and analyzed by an optical scanner for macrostructure analysis and scanning electron microscope (SEM) for microstructure analysis. The other part was put in 50\% diluted hydrochloric acid at $323 \mathrm{~K}$. The hydrochloric acid dissolved the eutectic aluminum phase, leaving the primary silicon phase (shape of flake) and the eutectic silicon phase (shape of powder). Then the primary silicon phase and the eutectic silicon phase were washed with deionized water, dried and separated by sieving with mesh size of $0.5 \mathrm{~mm}$. Then the boron and phosphorus contents in the primary silicon phase and the eutectic silicon phase were determined by ICP-OES.

\section{The boron and phosphorus distributions after the solidification of eutectic Al-Si melt}

The raw materials were prepared for $\mathrm{Al}-12.6 \% \mathrm{Si}$, which means that it is a eutectic Al-Si alloy. As summarized in Figure 1, the raw materials (approximate $250 \mathrm{~g}$ ) were put in an alumina crucible (I.D.: $55 \mathrm{~mm}$, and depth: $100 \mathrm{~mm}$ ). The alumina crucible was put in a resistance heating furnace with a proportional-integral-derivative (PID) controller to control temperature. The furnace was heated to $900 \mathrm{~K}$ ( $50 \mathrm{~K}$ above the eutectic temperature), and the alloy melted. After that, the melt was stirred by a quartz rod to mix it completely, and cooled to $830 \mathrm{~K}$ ( $20 \mathrm{~K}$ below the eutectic temperature) to solidify it into an ingot with the cooling rate shown in Table 2 . After that, the sample was furnace cooled to room temperature.

The solidified sample was put in 50\% diluted hydrochloric acid at $323 \mathrm{~K}$. The hydrochloric acid dissolved the eutectic aluminum phase, leaving the eutectic silicon phase (shape of powder). The eutectic silicon phase was washed with deionized water and dried. The boron and phosphorus contents in the eutectic silicon phase were determined by ICP-OES.

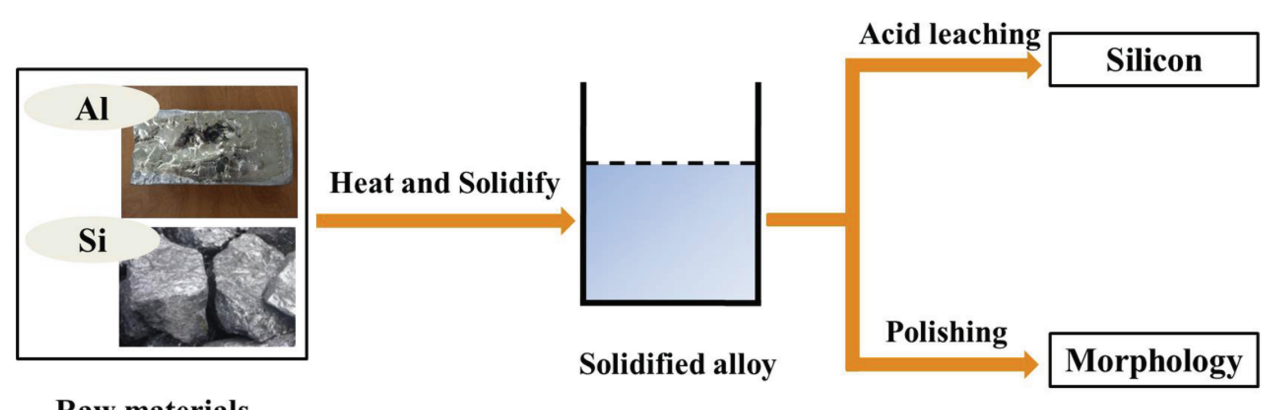

Raw materials

Figure 1: The schematic process of sample preparation and analysis. 
Table 2: Lists of samples.

\begin{tabular}{llcr}
\hline Experiment series & $\begin{array}{l}\text { Sample } \\
\text { number }\end{array}$ & Silicon ratio & Cooling rare $(\mathrm{mK} / \mathrm{s})$ \\
\hline Series 1 & No.1 & $20 \%$ & 47 \\
& No.2 & & 6.5 \\
Series 2 & No.3 & & 0.81 \\
& No.4 & $12.6 \%$ & 0.038 \\
\hline
\end{tabular}

\section{Results and discussion}

\section{The morphology of the primary silicon phase and the eutectic silicon phase}

Figure 2 shows the cross section of solidified sample No.2. It can be found that the primary silicon phases (C) locate at the edge of the image, and the eutectic $\mathrm{Al}-\mathrm{Si}$ matrix (A) and a cavity (B) can be seen.

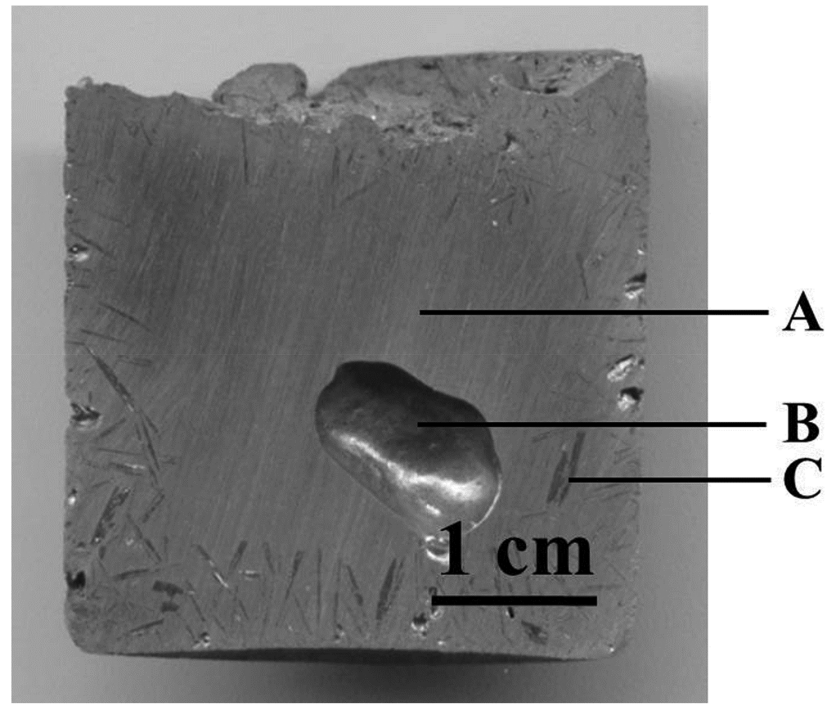

Figure 2: The cross section of solidified sample No.2.

In the hypereutectic Al-Si melt, when temperature is lower than liquidus, the primary silicon phase begins to grow. In this work, heat transports from melt into surrounding environment, and the temperature at the edge of the melt is lower than that in the middle of the melt. So the primary silicon phase first nucleates there and grows. According to the lever law and the Al-Si phase diagram [15], the amount of the primary silicon phase is constant. So when the primary silicon phase grows at the edge of the melt, and it cannot form in the middle of the melt.
When the primary silicon phase grows in the hypereutectic Al-Si melt, the composition of the remaining melt changes with liquidus. When the temperature drops at $850 \mathrm{~K}$ and the silicon ratio is $12.6 \%$, the eutectic reaction happens. In this reaction, the primary silicon phase and the eutectic silicon phase form, and they show as Al-Si matrix in macrostructure.

A cavity can be found in the image. At high temperature, Al-Si melt can absorb water vapor from environment and react with it, resulting in hydrogen atoms generated and dissolved in the melt. During the solidification of the alloy, the hydrogen atoms are rejected by the growing solid phase. If the hydrogen atoms cannot diffuse out of the melt during the solidification process, they will be trapped by the solid, forming hydrogen bubble. It appears as a cavity after cutting open the solidified ingot.

Figure 3(a) shows the SEM image of solidified sample No.2. It can be found primary silicon phase (D), eutectic silicon phase (E), eutectic aluminum phase (F) and white phases with needle like. To confirm the composition of the white phases, EDS analysis was conducted at Point 1, and the results are shown in Figure 3(b) and Table 3. It can be found that the white phase is an Al-Si-Fe-Mn phase. Because of the impurities in the raw materials, the $\mathrm{Al}-\mathrm{Si}-\mathrm{Fe}-\mathrm{Mn}$ intermediate compound phase forms in the solidification process.

Figure 4 shows the morphology of the primary silicon flakes and eutectic silicon powder. They are obtained after the hydrochloric treatment of solidified sample No.2. The primary silicon flake is corresponding to the primary silicon phase (D) in Figure 3(a), and the eutectic silicon phase is corresponding to eutectic silicon phase (E) in Figure 3(a). The eutectic aluminum phase and the Al-Si-Fe-Mn phase in Figure 3(a) were reacted with the hydrochloric acid.

As discussed before, the primary silicon phase grows in the temperature range between $971 \mathrm{~K}$ and $850 \mathrm{~K}$, and it grows into the shape of flake. The eutectic silicon phase forms at $850 \mathrm{~K}$ in the eutectic reaction. It does not have enough time to grow up and appears as powder after solidification.

\section{The boron and phosphorus distributions after the solidification of the hypereutectic Al-Si melt}

According to the result of microstructure in Figure 3, the primary silicon phase, the eutectic silicon phase, the eutectic aluminum phase and the Al-Si-Fe-Mn intermediate 

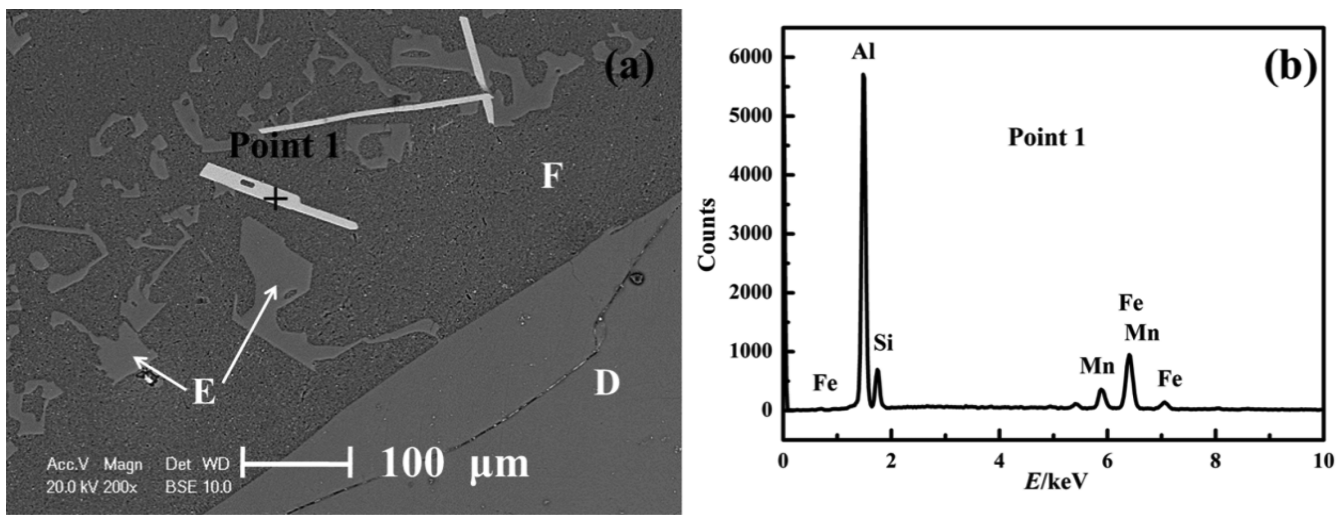

Figure 3: The SEM image of solidified sample No.2 and EDS image of Point 1 (a) SEM image of solidified sample No.2, (b) EDS image of Point 1.

Table 3: EDS quantitative analysis of point 1 located in Figure 3(a).

\begin{tabular}{lrrrrr}
\hline EDS analysis & \multicolumn{5}{c}{ Element (Atom \%) } \\
\hline & $\mathrm{Al}$ & $\mathrm{Si}$ & $\mathrm{Fe}$ & $\mathrm{Mn}$ & Total \\
Point 1 & 58.54 & 19.76 & 19.66 & 2.04 & 100 \\
\hline
\end{tabular}

compound phase form after the solidification of hypereutectic Al-Si melt in this work. The amount of Al-Si-Fe-Mn intermediate compound phase is small because of small Fe and $\mathrm{Mn}$ contents $(<1 \%)$ in the raw materials. Besides, according to the EDS result in Figure 3(b) and Table 3, The Al-Si-Fe-Mn intermediate compound phase does not contain boron and phosphorus. So the boron and phosphorus contents in the Al-Si-Fe-Mn intermediate compound are ignored in the following calculation.

We take boron as example to discuss the distribution of impurity element. The boron contents in the raw materials industrial grade aluminum and metallurgical grade silicon can be determined by ICP-OES, and they are shown in Table 1 . The boron contents in the primary silicon phase and the eutectic silicon phase after solidification of Al-Si melt can be confirmed by ICP-OES, and the values are shown in Table 4. The boron content in the eutectic aluminum phase can be obtained as following.

According to the Al-Si phase diagram [15] and level rule, the mass fractions of the primary silicon phase, the eutectic silicon phase and the eutectic aluminum phase after solidification of $\mathrm{Al}-20 \% \mathrm{Si}$ can be calculated as $0.00847,0.1153$, and 0.8 , respectively.

According to the conservation of mass, the boron distribution should follow eq. (1).

$$
0.00847 C_{P S i}+0.1153 C_{E S i}+0.8 C_{E A l}=0.2 C_{S i}+0.8 C_{A l}
$$

where, $C_{P S i}, C_{E S i}, C_{E A l}, C_{S i}$ and $C_{A l}$ are the boron contents in the primary silicon phase, the eutectic silicon phase,

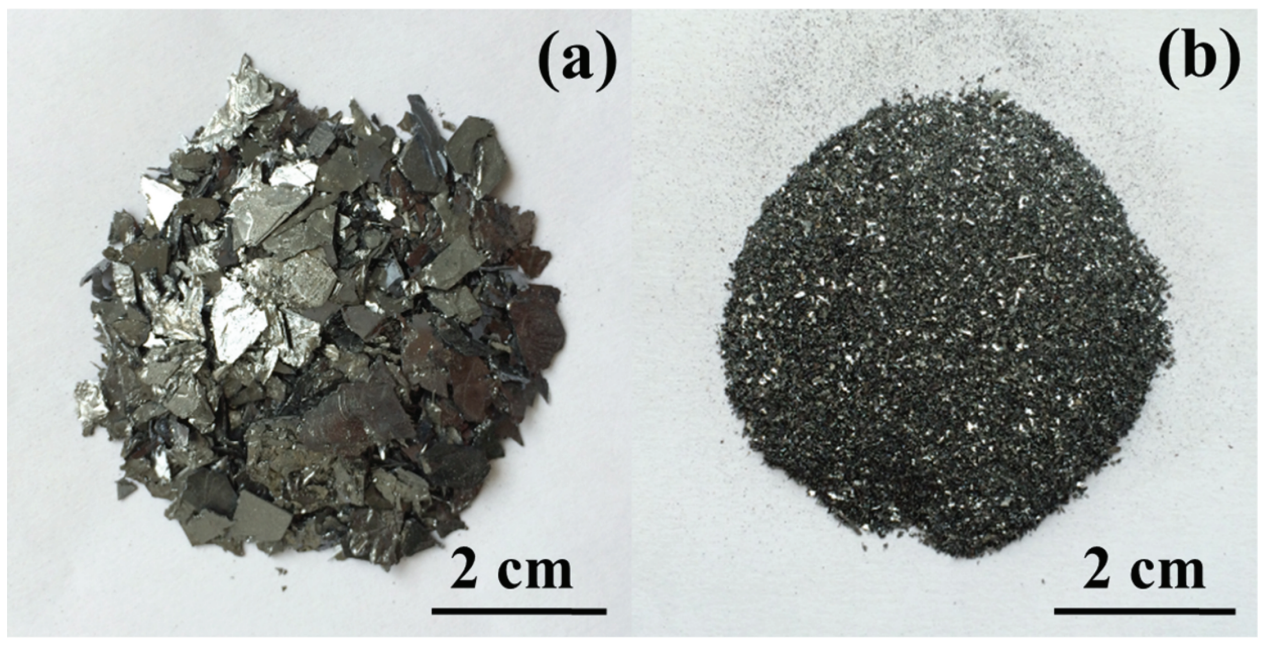

Figure 4: The morphology of primary silicon flakes and eutectic silicon powder, (a) primary silicon flakes, (b) eutectic silicon powder. 
Table 4: Boron and phosphorus contents in the primary silicon phase and the eutectic silicon phase.

\begin{tabular}{lrrrr}
\hline Sample number & \multicolumn{2}{c}{ Boron content (ppmw) } & Phosphorus content (ppmw) \\
\hline & In the primary silicon phase & In the eutectic silicon phase & In the primary silicon phase & In the eutectic silicon phase \\
Series 1 No.1 & 4.38 & 5.27 & 26.24 & 29.48 \\
Series 1 No.2 & 1.21 & 3.17 & 12.06 & 24.60 \\
Series 1 No.3 & 0.69 & 3.06 & 10.82 & 21.26 \\
\hline
\end{tabular}

the eutectic aluminum phase, the raw material metallurgical grade silicon and the raw material industrial grade aluminum respectively.

As discussed before, $C_{S i}$ and $C_{A l}$ are shown in shown in Table 1, and $C_{P S i} C_{E S i}$ are shown in Table 4. So $C_{E A l}$ can be obtained in eq. (2).

$$
C_{E A l}=\frac{\left(0.2 C_{S i}+0.8 C_{A l}\right)-\left(0.00847 C_{P S i}+0.1153 C_{E S i}\right)}{0.8}
$$

At last, the values of $C_{E A l}$ are all obtained.

Figure 5 shows the boron contents in the primary silicon phase, the eutectic silicon phase and the eutectic aluminum phase after the solidification of $\mathrm{Al}-20 \% \mathrm{Si}$ melt with different cooling rates (experiment series 1, sample No.1, 2, 3). It is obvious that it is a hypereutectic Al-Si solidification. The initial boron content in the melt is 7 ppmw which is obtained by $0.2 C_{S i}+0.8 C_{A l}$, and it is marked with dotted line in Figure 5.

Figure 5 indicates that the boron content in primary silicon phase is lowest, and the boron content in the eutectic aluminum phase is highest. It can be explained and discussed below.

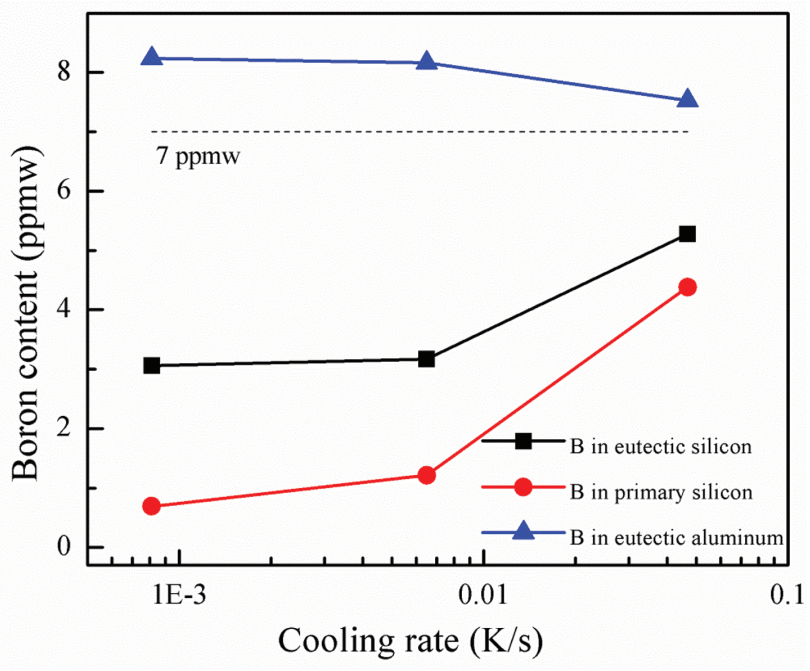

Figure 5: The boron contents in experiment series 1.
In the hypereutectic Al-Si melt, the primary silicon phase begins to grow when temperature falls below its liquidus, and the composition of the remaining melt changes with liquidus. At the same time boron atoms are excluded by the growing primary silicon phase, because its segregation coefficient is smaller than 1 . When the silicon ratio is $12.6 \%$ and the temperature is $577{ }^{\circ} \mathrm{C}$, the eutectic reaction happens, and the eutectic silicon phase and the eutectic aluminum phase form. At the time the boron content in the melt is higher than that in the primary silicon phase because boron atoms are enriched in the melt as discussed before. According to the work of M.M. Makhlouf [16], the eutectic silicon phase is the leading phase in the $\mathrm{Al}-\mathrm{Si}$ eutectic reaction. So boron atoms are rejected by the eutectic silicon phase, and they are enriched in the eutectic aluminum phase. So the boron content in the primary silicon phase is lowest, and the boron content in the eutectic aluminum phase is highest.

Figure 5 also shows that the cooling rate has great influence on the boron contents in the primary silicon phase, the eutectic silicon phase and the eutectic aluminum phase. It can be explained and discussed below.

As discussed before, when the primary silicon phase grows, boron atoms are rejected. They are enriched at the solid liquid interface, and diffuse into the remaining melt. The boron content in the primary silicon phase depends on the boron segregation coefficient and the boron content at the solid liquid interface as shown in eq. (3).

$$
C_{P S i}=k C_{\text {interface }}
$$

where, $C_{P S i}, k$ and $C_{\text {interface }}$ are the boron content in the primary silicon phase, the segregation coefficient of boron, and the boron content at the solid liquid interface respectively.

When the cooling rate is low, the enriched boron atoms at the solid liquid interface has enough time to diffuse into the remaining melt, and the boron content at the solid liquid interface decreases. According to eq. (3), the boron content in the primary silicon phase is low. 
In equilibrium solidification, the $\mathrm{Al}-\mathrm{Si}$ eutectic reaction happens at the temperature of $577^{\circ} \mathrm{C}$, but the equilibrium solidification cannot be satisfied in this work. When the cooling rare is low, the eutectic reaction happens slowly. As mentioned before, the eutectic silicon phase is the leading phase in the Al-Si eutectic reaction. So boron atoms are rejected by the eutectic silicon phase and enriched in the eutectic aluminum phase. At last, when the cooling rate is low, the boron content in eutectic silicon phases is low, and the boron content in the eutectic aluminum is high.

Figure 6 shows the phosphorus contents in the primary silicon phase, the eutectic silicon phase and the eutectic aluminum phase after the solidification of Al$20 \% \mathrm{Si}$ melt with different cooling rates (experiment series 1, sample No.1, 2, 3). The initial phosphorus content in the melt is 30.2 ppmw obtained by the same way as that of boron, and it is marked with dotted line in Figure 6. The distribution of phosphorus is similar to that of boron as discussed before.

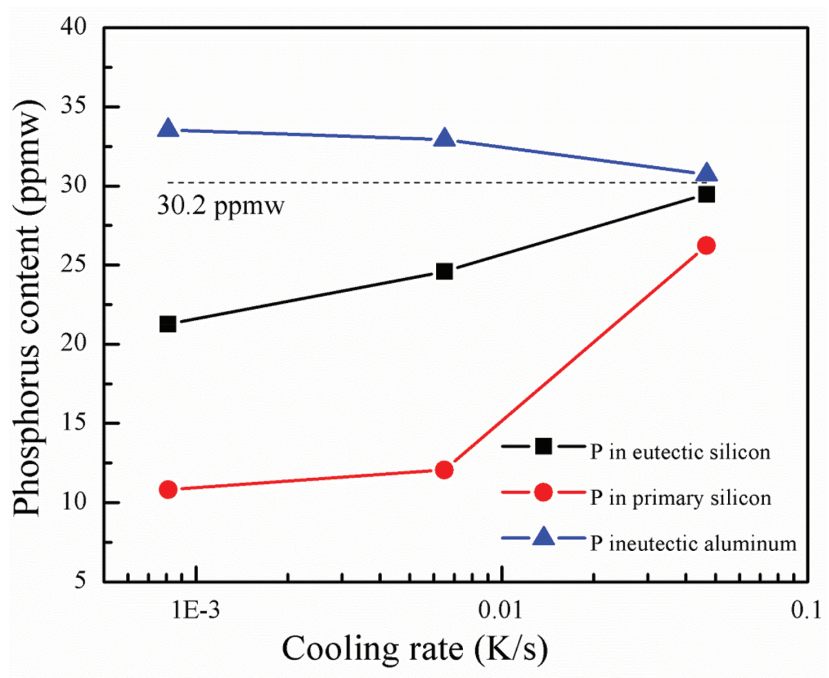

Figure 6: The phosphorus contents in experiment series 1 .

\section{The boron and phosphorus distributions after the solidification of the eutectic Al-Si melt}

After the solidification of the eutectic Al-Si melt (experiment series 2 No.4 in this work), the eutectic silicon phase and the eutectic aluminum phase form, and the primary silicon phase does not form. With the same calculation method mentioned before, the boron and phosphorus contents in the eutectic silicon phase and the eutectic aluminum phase can be obtained.

Figure 7(a) shows the boron contents in the eutectic silicon phase and the eutectic aluminum phase after the solidification of sample series 2 No.4. The boron contents in the eutectic silicon phase and the eutectic aluminum phase are 3.5 and 7.5 ppmw, respectively. The black dotted line shows the initial average boron content in the melt (value of $7 \mathrm{ppmw}$ ).

It can be found that the boron content in the eutectic silicon phase is lower than that in the eutectic aluminum phase. As discussed before, the eutectic silicon phase is the leading phase in the eutectic reaction. So the eutectic silicon phase can reject boron atoms, and has lower boron content after solidification. This result is in accordance with the result of the hypereutectic Al-Si melts solidification.

Figure 7(b) shows the phosphorus contents in the eutectic silicon phase and the eutectic aluminum phase after the solidification of sample series 2 No.4. The red dotted line shows the initial average phosphorus content in the melt ( $32 \mathrm{ppmw})$. The phosphorus distribution in the eutectic silicon phase and the eutectic aluminum phase is similar to that of boron as discussed before.

\section{Conclusions}

The hypereutectic Al-Si melts and the eutectic Al-Si melt were solidified to study the boron and phosphorus distributions in the primary silicon phase, the eutectic silicon phase and the eutectic aluminum phase during $\mathrm{Al}-\mathrm{Si}$ solvent refining. In hypereutectic Al-Si melt, the primary silicon phase can reject boron and phosphorus atoms, and it has lowest boron and phosphorus contents after solidification. In the eutectic reaction, the eutectic silicon phase is the leading phase, and it can reject boron and phosphorus atoms. The eutectic aluminum phase has highest boron and phosphorus contents after solidification.

Acknowledgements: This work was supported by Project of Science and Technology Department of Henan Province (No. 172102210460), Doctoral Scientific Research Foundation of 

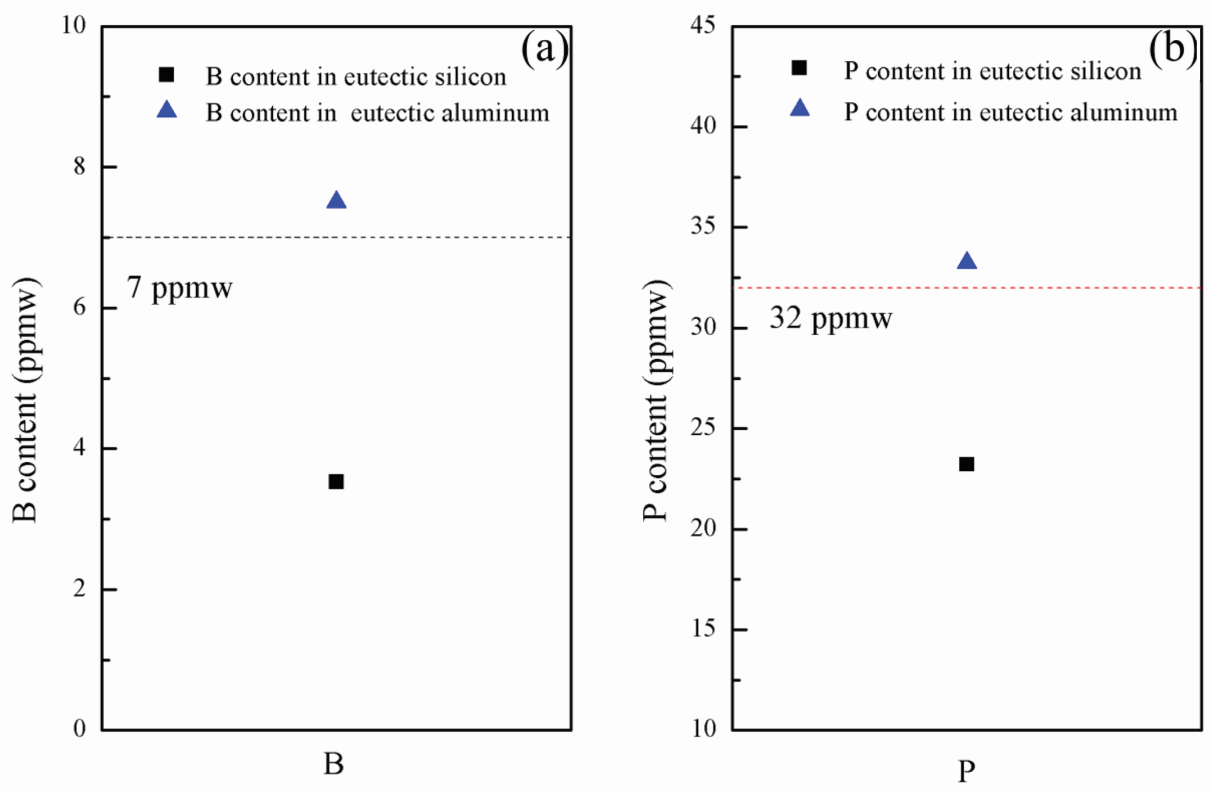

Figure 7: The boron and phosphorus distributions in sample series 2 No.4, (a) boron distribution, (b) phosphorus distribution.

Xinyang Normal University, and Nanhu Scholars Program for Yong Scholars of XYNU.

\section{References}

[1] A. Saito, A. Sakurai and H. Habuka, J. Cryst. Growth, 468 (2017) 204-207.

[2] F.A. Trumbore, Bell Syst. Techn. J., 39 (1960) 205-233.

[3] T. Yoshikawa and K. Morita, Metall. Mater. Trans. B, 36 (2005) 731-736.

[4] T. Yoshikawa and K. Morita, J. Cryst. Growth, 311 (2009) 776-779.

[5] T. Yoshikawa and K. Morita, Sci. Techn. Advan. Mater., 4 (2003) 531-537.

[6] K. Tang, E.J. Øvrelid, G. Tranell and M. Tangstad, Mater. Trans., 50 (2009) 1978-1984.
[7] L.X. Zhao, Z. Wang, Z.C. Guo and C.Y. Li, Tran. Nonferrous Met. Soc. China, 21 (2011) 1185-1192.

[8] L. Hu, Z. Wang, X.Z. Gong, Z.C. Guo and H. Zhang, Metall. Mater. Trans. B, 44 (2013) 828-836.

[9] X. Gu, X.G. Yu and D.R. Yang, Sep. Purif. Technol., 77 (2011) 33-39.

[10] P.P. Wang, H.M. Lu and Y.S. Lai, J. Cryst. Growth, 390 (2014) 96-100.

[11] J.W. Li, Z.C. Guo, H.Q. Tang and J.C. Li, High Temp. Mater. Proc, 32 (2013) 503-510.

[12] Y.L. Li, J. Chen, B.Y. Ban, T.T. Zhang and S.Y. Dai, High Temp. Mater. Proc., 34 (2015) 43-49.

[13] B.B. Ban, X.L. Bai, J.W. Li, J. Chen and S.Y. Dai, J. Alloy Compd., 685 (2016) 604-609.

[14] X.L. Bai, B.Y. Ban, J.W. Li, Z.Q. Fu, Z.J. Peng, C.B. Wang and J. Chen, Sep. Purif. Technol., 174 (2017) 345-351.

[15] J.L. Murray, A. J. McAlister, 5 (1984) 74-84.

[16] M.M. Makhlouf and H.V. Guthy, J. Light Met., 1 (2001) 199-218. 